Research Article

\title{
Reliable Positioning Algorithm Using Two-Stage Adaptive Filtering in GPS-Denied Environments
}

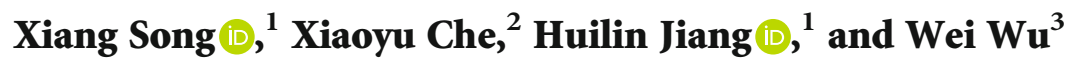 \\ ${ }^{1}$ School of Electronic Engineering, Nanjing Xiaozhuang University, Nanjing 211171, China \\ ${ }^{2}$ National Engineering Research Center of Road Maintenance Technologies, Beijing 100095, China \\ ${ }^{3}$ School of Instrument Science and Engineering, Southeast University, Nanjing 210096, China
}

Correspondence should be addressed to Xiang Song; sx2190105@163.com

Received 3 January 2020; Revised 25 September 2020; Accepted 30 September 2020; Published 24 October 2020

Academic Editor: Xavier Vilanova

Copyright (c) 2020 Xiang Song et al. This is an open access article distributed under the Creative Commons Attribution License, which permits unrestricted use, distribution, and reproduction in any medium, provided the original work is properly cited.

To overcome the disadvantages of RFID application for outdoor vehicle positioning in completely GPS-denied environment, a fusion vehicle positioning strategy based on the integration of RFID and in-vehicle sensors is proposed. To obtain higher performance, both preliminary and fusion positioning algorithms are studied. First, the algorithm for preliminary positioning is developed in which only RFID is adopted. In the algorithm, through using the received signal strength, range from RFID tags to the reader is estimated by implementing the extreme learning machine algorithm, and then, the first-level adaptive extended Kalman filter (AEKF) which can accommodate the uncertainties in the observation noise description of RFID is employed to compute the vehicle's location. Further, to compensate the deficiencies of preliminary positioning, the in-vehicle sensors are introduced to fuse with RFID. The second-level adaptive decentralized information filtering (ADIF) is designed to achieve fusion. In the implementation process of ADIF, the improved vehicle motion model is established to accurately describe the motion of the vehicle. To isolate the RFID failure and fuse multiple observation sources with different sample rates, instead of the centralized EKF, the decentralized architecture is employed. Meanwhile, the adaptive rule is designed to judge the effectiveness of preliminary positioning result, deciding whether to exclude RFID observations. Finally, the proposed strategy is verified through field tests. The results validate that the proposed strategy has higher accuracy and reliability than traditional methods.

\section{Introduction}

Driven by evolution of wireless communication technologies, indoor wireless positioning systems develop rapidly [1-3]. Many mature location methods have been proposed and widely adopted to obtain location information of the object in indoor environment [4]. Among them, radio frequency identification (RFID) attracts widespread attention [5]. Since the in-depth study of RFID positioning technology in indoor environment, technologies for outdoor vehicle positioning by RFID begin to be studied [6]. RFID could separately provide location information for vehicles, not needing the help of other sensors. However, due to severe nonlinearities during the operation process of vehicles, simply using RFID could not achieve high positioning performance in accuracy and output frequency. In addition, by RFID, only position infor- mation could be provided, lacking velocity information which is also important to the vehicle.

For vehicle localization, the global positioning system (GPS) is the most widespread approach [7]. However, a crucial problem of GPS is its performance degradation in GPS-denied environment due to the signal blockage or multipath interference [8]. In order to mitigate the performance degradation of GPS, a combination of the inertial navigation system (INS) [9] or dead reckoning (DR) [10] with GPS is often utilized. INS or DR takes advantage of the previous position of vehicles to estimate their current position. Due to the measurement biases of inertial sensors and the characteristics of the integration process [11], overtime, the positioning error of INS or DR accumulates such that the accuracy cannot be trusted for long no-GPS periods. Other in-vehicle sensors [12] are used to compensate for these 
errors. However, the compensation effect is poor in completely GPS-denied environments, as the accumulative errors of low-cost in-vehicle sensors cannot be compensated without the position observation. Although the unscented Kalman filter (UKF), cubature Kalman filter (CKF) [13, 14], and strong tracking Kalman filter and other nonlinear filtering algorithms have achieved good results, they are still limited due to the lack of position observation information in the long-term failure environment of GPS. For example, an adaptive Kalman filter framework based on Sage-Husa and strong tracking filtering algorithms has been proposed in [14], which was implemented on MEMS-IMU and the GPS for the case of a ground vehicle and achieved good positioning performance.

Thus, it can be seen from the above discussion that RFID could provide location information of the vehicle in completely GPS-denied environments. Meanwhile, DR technology is widely used for vehicle positioning in GPS-denied environments which uses the driving direction and speed to reckon the position of the vehicle [15]. Self-contained feature is an advantage of DR. However, for numerical integration processing, this method suffers from serious accumulative integration errors when GPS signal is not available for quite a while, and these large errors are strongly time correlated, which could cause rapid performance degradation due to the lack of position observations.

RFID and DR are complementary. Through combining their advantages, better positioning performance in completely GPS-denied environments could be achieved. To compensate the deficiencies of RFID, in-vehicle sensors could be introduced to combine with RFID [6]. However, to the authors' best knowledge, there has been little relevant research on the topic of fusion positioning specialized for vehicle by fusing RFID and in-vehicle sensors.

As mentioned above, RFID could provide the position observation to correct the accumulative integration errors of DR, and DR could provide motion information of the vehicle to improve the positioning accuracy and output frequency of RFID. However, many problems still exist in the process of integration. The main reason is that in outdoor application, the layout density of RFID tags could not be as big as that in indoor environment due to the cost considerations, leading to tags which could be detected are not enough, thus resulting in filtering divergence. The divergence may decrease the performance of positioning system obviously. To solve this problem, the authors have studied and compared many filtering methods, such as extended Kalman filter (EKF), unscented Kalman filter (UKF), particle filter (PF), strong tracking EKF (STEKF), and federated filter and interactive multiple model filter (IMM), proposing that the combination of the methodology of decentralized filtering [16] with the adaptive filtering [17] may provide a viable solution to solve this problem.

In this paper, a positioning strategy for the vehicle in completely GPS-denied environments is proposed. This strategy employs two-step approaches, namely, the preliminary positioning only utilizing RFID, then further fusion positioning. In preliminary positioning, the received signal strength (RSS) of RFID is employed as an indicator to preliminary compute the vehicle's location. The main contributions can be listed as follows:

(1) An extreme learning machine (ELM) algorithm is designed to estimate the ranges from the tags to the vehicle based on RSS. Compared to the traditional propagation model method [5], the ELM algorithm has advantages such as high precision, high generalization ability, and strong adaptability for different environments, which could obviously improve the accuracy of preliminary positioning

(2) A two-level filtering method is designed to achieve the preliminary and fusion positioning

(i) After the estimation of the ranges from the vehicle to the RFID tags, the first-level filtering algorithm is employed to achieve preliminary positioning. Rather than the commonly used EKF, the innovation adaptive estimation- (IAE-) based adaptive EKF (AEKF) is adopted to accommodate the uncertainties in the observation noise description of RFID and then improve the positioning performance

(ii) A novel adaptive decentralized information filtering (ADIF) algorithm is proposed to realize the multisensor fusion positioning. To overcome the deficiency of RFID positioning, in-vehicle DR motion sensors are introduced to fuse with preliminary positioning results for high accuracy and output frequency, and the second-level filter is employed to achieve the fusion. However, the standard EKF has some defects for vehicle positioning, so the ADIF algorithm is proposed to replace the standard EKF in second-level filter. Compared with the centralized filter, the decentralized information filtering algorithm is convenient to isolate the RFID failure and fuse multiple observation sources with different sample rates. Moreover, the adaptive approach is employed to judge the effectiveness of preliminary positioning results and then decide whether to exclude the RFID observations when RFID is unavailable

The remainder of the paper proceeds as follows. The methodology is given in Section 2. Section 3 presents the RFID positioning algorithm for vehicle. Section 4 is devoted to the fusion positioning algorithm. Section 5 shows the experimental results and analysis. Section 6 gives the conclusion.

\section{Methodology}

The proposed strategy is shown in Figure 1. There are three main components, i.e., the multisensor part, the preliminary positioning part, and the fusion positioning algorithm part. Meanwhile, the range estimation algorithm is developed before preliminary positioning and the improved vehicle motion model is established in the fusion process. 


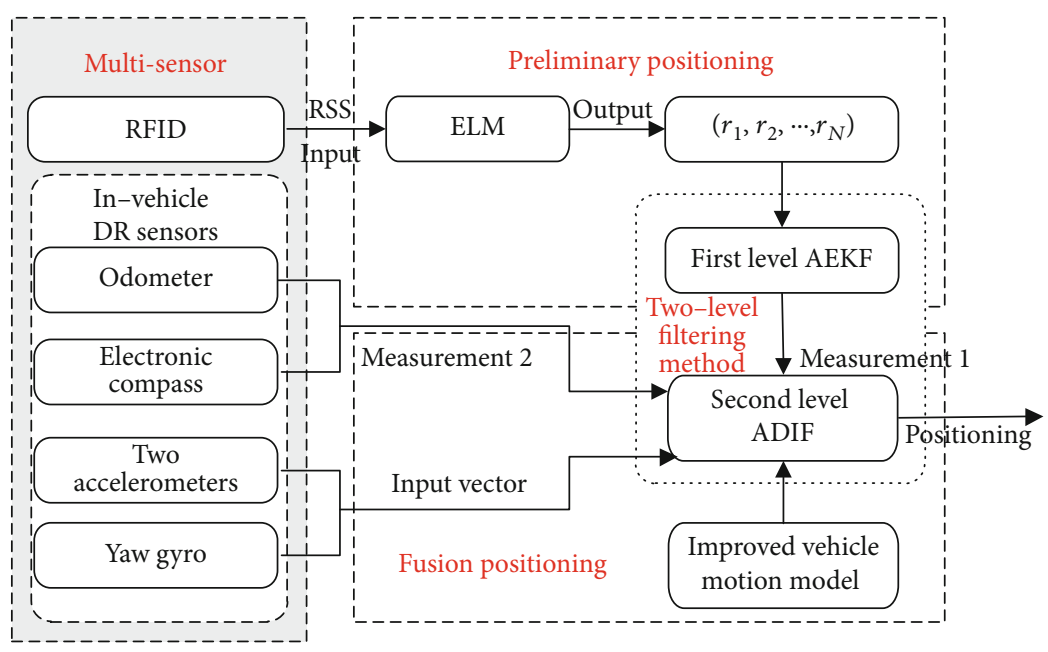

FIgURE 1: Proposed vehicle positioning strategy.

The multisensor part includes the accelerometer, gyro, odometer, electronic compass, and active RFID devices which contain one reader and some tags.

The two-level filtering method is proposed to achieve the preliminary and fusion positioning, i.e., AEKF for the first level and ADIF for the second level.

Based on the existing studies of indoor positioning, the RFID technology is considered to achieve preliminary positioning. In this paper, the RSS is employed to compute the vehicle's position because no additional hardware is needed. The preliminary algorithm can be divided into two stages, namely, the range calculation from RFID tags to RFID reader, and then, the first-level AEKF is employed to preliminary locate the vehicle's position. The ELM algorithm, which has been proved to be effective for modeling the systems with nonlinearity and parametric uncertainties, is proposed to identify the range. Rather than the conventional propagation model method which is the main approach for distance estimation in indoor positioning based on RSS [2], the ELM could achieve better performance since the accuracy of the propagation method may be influenced by environments. Through estimation of the innovation vector, the AEKF adaptively adjusts the observation noise covariance vector according to the changes of observation noise of RFID, which can prevent filter divergence and improve the positioning accuracy.

Further, utilizing the second-level ADIF, fusion positioning is carried out by integrating low-cost DR sensors and the results from the first-level AEKF. Owing to the DR sensors, the further positioning algorithm may enhance the accuracy and output frequency. Meanwhile, to describe the vehicle motion more accurately, the improved vehicle motion model is established in the fusion process. In the actual implementation of second-level filtering fusion, the ADIF is applied. For vehicles, there are more advantages of ADIF than traditional EKF due to the decentralized architecture: (1) convenient to fuse different sensors with different sampling rates, for example, the sampling rate of RFID is always $1 \mathrm{~Hz}$, lower than that of DR in-vehicle sensors, and (2) convenient to exclude the failure information when preliminary position- ing results are unavailable; moreover, the adaptive rule is proposed to detect the RFID failure.

\section{Preliminary Positioning}

3.1. Range Estimation Algorithm. It seems that the first challenge is to model the nonlinear correlation between the RSS and the range. Theoretically, under ideal environments, Friis transmission equation (5) can be applied. However, due to the impact of environment, this model does not satisfy the requirement of real-world applications. This paper proposes an ELM algorithm to model the relationship. Compared to the propagation model, there are many advantages in the proposed ELM algorithm, such as high precision, high generalization ability, and strong adaptability for different environments, which could improve the positioning performance significantly. The input of ELM is RSS, and the output is the range. The training data is collected through experiments in different situations. The training process is off-line, and the trained ELM [18] is used to estimate the range online.

Assuming that $\left\{\left(\mathbf{x}_{i}, \mathbf{y}_{i}\right) \mid \mathbf{x}_{i} \in \mathbf{R}^{n}, \mathbf{y}_{i} \in \mathbf{R}^{m}, i=1, \cdots, N\right\}$ is a training set, $f(x)$ is the activation function, and $\tilde{N}$ is the hidden neuron number, the ELM training algorithm can be summarized as follows:

(i) Randomly assign $\mathbf{w}_{i}$ (the weight vector connecting the $i$ th hidden neuron with the input neurons) and $b_{i}$ (the threshold of the $i$ th hidden neuron), $i=1,2,3 \cdots \tilde{N}$

(ii) Calculate the hidden layer output matrix $\mathbf{B}$

$$
\begin{aligned}
& \mathbf{B}\left(\mathbf{w}_{1}, \cdots \mathbf{w}_{\tilde{N}}, b_{1}, \cdots, b_{\tilde{N}}, \mathbf{x}_{1}, \cdots, \mathbf{x}_{N}\right) \\
& =\left[\begin{array}{ccc}
f\left(\mathbf{w}_{1} \cdot \mathbf{x}_{1}+b_{1}\right) & \cdots & f\left(\mathbf{w}_{\tilde{N}} \cdot \mathbf{x}_{1}+b_{\tilde{N}}\right) \\
\vdots & \ddots & \vdots \\
f\left(\mathbf{w}_{1} \cdot \mathbf{x}_{N}+b_{1}\right) & \cdots & f\left(\mathbf{w}_{\tilde{N}} \cdot \mathbf{x}_{N}+b_{\tilde{N}}\right)
\end{array}\right]_{N \times \tilde{N}},
\end{aligned}
$$

where $\mathbf{w}_{i} \cdot \mathbf{x}_{j}$ denotes the inner product of $\mathbf{w}_{i}$ and $\mathbf{x}_{j}$ 
(iii) Calculate the output weight $\widehat{\boldsymbol{\beta}}$

$$
\widehat{\boldsymbol{\beta}}=\mathbf{B}^{+} \mathbf{Y},
$$

where $\mathbf{B}^{+}$is the Moore-Penrose generalized inverse of matrix $\mathbf{B}, \mathbf{Y}=\left[\mathbf{y}_{1}, \cdots, \mathbf{y}_{N}\right]^{T}, \widehat{\boldsymbol{\beta}}=\left[\beta \wedge_{1}, \cdots, \beta \wedge_{\tilde{N}}\right]^{T}, \widehat{\boldsymbol{\beta}}_{i}$ is the weight vector connecting the $i$ th hidden neuron with the output neurons

After training, the ELM could be used as standard Singlehidden Layer Feedforward Neural Network (SLFN) with activation function $f(x)$ and $\tilde{N}$ hidden neurons. The mathematical model is

$$
\mathbf{o}_{j}=\sum_{i=1}^{\tilde{N}} \widehat{\beta}_{i} f\left(\mathbf{w}_{i} \cdot \mathbf{x}_{j}+b_{i}\right) j=1, \cdots, N
$$

where $\mathbf{o}_{j}$ is the output value of $j$ th input sample $\mathbf{x}_{j}$.

The special solution $\widehat{\boldsymbol{\beta}}=\mathbf{B}^{+} \mathbf{Y}$ is one of the least-squares solutions of a general linear system $\mathbf{B} \boldsymbol{\beta}=\mathbf{Y}$, which means that the smallest training error can be reached, shown as

$$
\|\mathbf{B} \widehat{\boldsymbol{\beta}}-\mathbf{Y}\|=\left\|\mathbf{B B}^{+} \mathbf{Y}-\mathbf{Y}\right\|=\min _{\boldsymbol{\beta}}\|\mathbf{B} \boldsymbol{\beta}-\mathbf{Y}\| .
$$

In this paper, we choose the most commonly used radial basis function as the activation function.

After off-line training, equation (3) could be used to estimate the range between tag and reader based on measured RSS online.

3.2. Preliminary Algorithm. The RFID reader is placed on the vehicle roof. Its position is considered as the vehicle position. The RFID tags are put on both sides of the road with exact known position.

The second step is how to calculate the position of the vehicle according to the estimated ranges. For indoor location estimation, the multilateration method is the most popular approach [19]. However, due to the adverse phenomena such as fading, absorption, and multipath of propagation, the calculated ranges usually contain a great error and then cause larger positioning error. To address this, the first-level filtering algorithm is developed to determine the position of the vehicle by using the ranges.

It has been shown that the standard EKF depends largely on the accuracy of the measurement noise vector. Incorrect a priori knowledge of observation noise covariance matrix may lead to filter divergence and performance degradation. However, the adverse phenomena as discussed above may affect the observation noise seriously. Therefore, the IAE-based AEKF [20] is adopted to achieve preliminary positioning in this section.

Assumed that $i$ tags with known coordinates $\left(x_{i}, y_{i}\right)$ are detected at time $k$, and the ranges from these tags to the vehicle, $r_{1}(k), r_{2}(k), \cdots, r_{N}(k)$, can be calculated by using (3). The discretized state equation and measurement equation can be described as

$$
\mathbf{X}_{\mathrm{R}}(k)=\mathbf{A}_{\mathrm{R}} \mathbf{X}_{\mathrm{R}}(k-1)+\mathbf{W}_{\mathrm{R}}(k-1),
$$

$$
\mathbf{Z}_{\mathrm{R}}(k)=\mathbf{h}_{\mathrm{R}}\left(\mathbf{X}_{\mathrm{R}}(k)\right)+\mathbf{V}_{\mathrm{R}}(k),
$$

where $k$ is the discrete-time step; $\mathbf{X}_{\mathrm{R}}(k)=[e(k) n(k)]^{\prime}$ indicates the state vector, $e$ and $n$ are the east and north positions of the vehicle; $\mathbf{A}_{R}=\left[\begin{array}{ll}1 & 0 \\ 0 & 1\end{array}\right]$ is the state transition matrix; $\mathbf{W}_{R}$ and $\mathbf{V}_{R}$ are the system and measurement noise and their covariance matrices are $\mathbf{Q}_{\mathrm{R}}$ and $\mathbf{R}_{\mathrm{R}}$, respectively; $\mathbf{Z}_{\mathrm{R}}(k)=$ $\left[r_{1}(k) \cdots r_{i}(k)\right]^{\prime}$ is the observation vector and the observation function is $\mathbf{h}_{\mathrm{R}}(k)=\left[\begin{array}{lll}h_{1}(k) & \cdots & h_{i}(k)\end{array}\right]^{\prime}, \quad h_{i}(k)=$ $\sqrt{\left(e(k)-x_{i}(k)\right)^{2}+\left(n(k)-y_{i}(k)\right)^{2}}+n_{r_{i}}, \quad n_{r_{i}} \quad$ denotes the observation noise vector of ranges.

The principle of IAE-based adaptive EKF is that the innovation vector from historical epochs can be used to evaluate the measurement precision of the present epoch.

The standard EKF can be described as follows:

$\widehat{\mathbf{X}}_{\mathrm{R}}(k, k-1)=\mathbf{A}_{\mathrm{R}} \widehat{\mathbf{X}}_{\mathrm{R}}(k-1)$,

$\mathbf{P}_{\mathrm{R}}(k, k-1)=\mathbf{A}_{\mathrm{R}}(k, k-1) \mathbf{P}_{\mathrm{R}}(k-1) \mathbf{A}_{\mathrm{R}}^{\prime}(k, k-1)+\mathbf{Q}_{\mathrm{R}}(k-1)$,

$$
\begin{aligned}
\mathbf{K}_{\mathrm{R}}(k)= & \mathbf{P}_{\mathrm{R}}(k, k-1) \cdot \mathbf{H}_{1}^{\prime}(k) \\
& \cdot\left[\mathbf{H}_{\mathrm{R}}(k) \mathbf{P}_{\mathrm{R}}(k, k-1) \mathbf{H}_{\mathrm{R}}^{\prime}(k)+\mathbf{R}_{\mathrm{R}}(k)\right]^{-1}, \\
\widehat{\mathbf{X}}_{\mathrm{R}}(k)= & \widehat{\mathbf{X}}_{\mathrm{R}}(k, k-1)+\mathbf{K}_{\mathrm{R}}(k)\left[\mathbf{Z}_{\mathrm{R}}(k)-\mathbf{h}_{\mathrm{R}} \widehat{\mathbf{X}}_{\mathrm{R}}(k, k-1)\right],
\end{aligned}
$$

$$
\mathbf{P}_{\mathrm{R}}(k)=\left[\mathbf{I}-\mathbf{K}_{\mathrm{R}}(k) \cdot \mathbf{H}_{\mathrm{R}}(k)\right] \mathbf{P}_{\mathrm{R}}(k, k-1),
$$

where $\mathbf{I}$ is an identity matrix and $\mathbf{H}_{\mathrm{R}}$ is the Jacobian matrix of the measurement function $\mathbf{h}_{\mathrm{R}}(\cdot)$ with respect to $\mathbf{X}_{\mathrm{R}}$.

The adaptive rule in the EKF is that the measurement covariance should be tuned to make innovation message consistent with their theoretical covariance. The innovation can be defined as

$$
\mathbf{v}_{\mathrm{R}}(k)=\mathbf{Z}_{\mathrm{R}}(k)-\mathbf{h}_{\mathrm{R}}\left(\widehat{\mathbf{X}}_{\mathrm{R}}(k, k-1)\right),
$$

where $\mathbf{v}_{\mathrm{R}}(k)$ is considered as the difference between the real observations and its computed values, and the theoretical covariance of $\mathbf{v}_{\mathrm{R}}(k)$ is

$$
\mathbf{C}_{\mathbf{v}}(k)=\mathbf{H}_{\mathrm{R}}(k) \mathbf{P}_{\mathrm{R}}(k, k-1) \mathbf{H}_{\mathrm{R}}^{\prime}(k)+\mathbf{R}_{\mathrm{R}}(k) .
$$

Meanwhile, the actual covariance of $\mathbf{v}_{\mathrm{R}}(k)$ can be computed through averaging the previous innovation sequence over a moving window of size $m$ at epoch $k$ :

$$
\widehat{\mathbf{C}}_{\mathbf{v}}(k)=\frac{1}{m} \sum_{i=1}^{m} \mathbf{v}_{\mathrm{R}}(k-1) \mathbf{v}_{\mathrm{R}}{ }^{\prime}(k-1),
$$


where $\widehat{\mathbf{C}}_{\mathbf{v}}(k)$ is the estimation variance-covariance of innovation. Take (14) into (13), we get the adaptive measurement noise covariance as follows:

$$
\widehat{\mathbf{R}}_{\mathrm{R}}(k)=\widehat{\mathbf{C}}_{\mathbf{v}}(k)-\mathbf{H}_{\mathrm{R}}(k) \mathbf{P}_{\mathrm{R}}(k, k-1) \mathbf{H}_{\mathrm{R}}^{\prime}(k) .
$$

By replacing $\mathbf{R}_{\mathrm{R}}(k)$ with $\widehat{\mathbf{R}}_{\mathrm{R}}(k)$ in (9), the IAE-based adaptive EKF is realized.

Since only the RFID is employed to achieve vehicle positioning, this algorithm could only provide the position of vehicle; accuracy and positioning frequency are lower for the outdoor application, i.e., the output frequency is always less than $1 \mathrm{~Hz}$.

\section{Fusion Positioning}

To improve the performance of preliminary positioning algorithm, the fusion positioning algorithm is developed.
Some DR motion sensors are introduced to combine with the RFID information; the second-level ADIF is designed to achieve the fusion positioning.

4.1. Vehicle Motion Model. To describe the typical vehicle motion, the improved vehicle motion model is established. The state vector is

$$
\mathbf{X}(k)=\left[\begin{array}{lllll}
e(k) & n(k) & v_{e}(k) & v_{n}(k) & \psi(k)
\end{array}\right]^{\prime},
$$

where $v_{e}(k)$ and $v_{n}(k)$ denote the east and north velocity and $\psi(k)$ refers to the yaw angle.

To describe the wide driving-maneuver motion range, the longitudinal acceleration $a_{x}$, the lateral accelerations $a_{y}$, and the yaw rate $\omega_{z}$, measured by the accelerometers and yaw gyro, are introduced as system input vector in the vehicle motion model. The improved vehicle motion model [21] equation is shown as follows:

$$
\mathbf{X}(k, k-1)=\mathbf{f}(\mathbf{X}(k-1), \mathbf{U}(k))=\left[\begin{array}{c}
e(k-1)+v_{e}(k-1) T+\frac{1}{2}\left[a_{x} \cos \psi(k-1)-a_{y} \sin \psi(k-1)\right] T^{2} \\
v_{e}(k-1)+\left[a_{x} \cos \psi(k-1)-a_{y} \sin \psi(k-1)\right] T \\
n(k-1)+v_{n}(k-1) T+\frac{1}{2}\left[a_{x} \sin \psi(k-1)+a_{y} \cos \psi(k-1)\right] T^{2} \\
v_{n}(k-1)+\left[a_{x} \sin \psi(k-1)+a_{y} \cos \psi(k-1)\right] T \\
\psi(k-1)+\omega_{z}
\end{array}\right],
$$

where $T$ is the sampling interval; $\mathbf{f}(\cdot)$ denotes the process function of the improved vehicle motion model; $\mathbf{U}=$ $\left[\begin{array}{lll}a_{x} & a_{y} & \omega_{z}\end{array}\right]^{\prime}$ is the input vector. Due to the input vector, the model could accommodate the typical motion patterns of vehicle such as acceleration, deceleration, turn motion, or lane change.

4.2. Measurement Model. From Figure 1, it could be seen that the measurement vector $\mathbf{Z}$ comes from RFID and in-vehicle DR sensors, respectively, i.e., the estimated vehicle's coordinates, $x_{\mathrm{R}}$ and $y_{\mathrm{R}}$, estimated by the first-level AEKF, the observed vehicle velocity $v_{o}$, and yaw angle $\psi_{c}$ measured by odometer and compass. The measurement equation of preliminary positioning and DR sensors is established as

$$
\begin{aligned}
& \mathbf{Z}_{\mathrm{R}}=\mathbf{H}_{\mathrm{R}} \mathbf{X}(k)+\mathbf{n}_{\mathrm{R}}=\left[\begin{array}{ll}
1 & 0 \\
0 & 1
\end{array}\right] \mathbf{X}(k)+\left[\begin{array}{c}
n_{p e} \\
n_{p n}
\end{array}\right], \\
& \mathbf{Z}_{\mathrm{V}}=\mathbf{h}_{\mathrm{V}}[\mathbf{X}(k)]+\mathbf{n}_{\mathrm{V}}=\left[\begin{array}{c}
v_{e}(k) \cos \psi(k)+v_{n}(k) \sin \psi(k)+n_{v} \\
\psi(k)+n_{\psi}
\end{array}\right],
\end{aligned}
$$

where $\mathbf{Z}_{\mathrm{R}}=\left[\begin{array}{ll}x_{\mathrm{R}} & y_{\mathrm{R}}\end{array}\right]^{\prime}$ and $\mathbf{Z}_{\mathrm{V}}=\left[\begin{array}{ll}v_{\mathrm{O}} & \psi_{\mathrm{c}}\end{array}\right]^{\prime}$ denote the observation vectors from preliminary positioning and DR in-vehicle sensors. $\mathbf{H}_{\mathrm{R}}$ and $\mathbf{h}_{\mathrm{V}}$ represent the corresponding observation matrix and function. $\mathbf{n}_{\mathrm{R}}=\left[\begin{array}{ll}n_{p e} & n_{p n}\end{array}\right]^{\prime}$ and $\mathbf{n}_{\mathrm{V}}=\left[\begin{array}{ll}n_{v} & n_{\psi}\end{array}\right]^{\prime}$ are the corresponding noise vectors.

4.3. ADIF Algorithm. In practice, there are two challenges that should be considered:

(i) RFID and in-vehicle sensors have different sampling rates

(ii) Preliminary positioning algorithm fails to provide accurate positioning information if there is no RSS signal or information is valid due to the phenomenon such as communication failure, multipath interference, or no enough number of tags can be detected. The improper data can easily influence the fusion

The widely used EKF is difficult to deal with the above two aspects in the fusion process due to its centralized filtering architecture. To solve this problem, the decentralized information filter provides us a viable solution. Therefore, in this paper, ADIF is designed to realize fusion positioning. ADIF is a decentralized information filtering algorithm with some adaptive rules which can handle the 
above problems. For the vehicle, it has the following advantages over commonly used EKF:

(i) The decentralized architecture can combine the information with different sampling rates

(ii) The adaptive rules can quickly judge the failure information from the preliminary positioning results and exclude the failure information due to the decentralized architecture

For the model illustrated by (16)-(19), the execution of ADIF contains two stages:

(1) Time update:

$$
\begin{aligned}
\widehat{\mathbf{X}}(k, k-1)= & \mathbf{f}(\overline{\mathbf{X}}(k-1), \mathbf{U}(k)), \\
\mathbf{P}(k, k-1)= & \mathbf{A}(k, k-1) \overline{\mathbf{P}}(k-1) \mathbf{A}^{\prime}(k, k-1) \\
& +\mathbf{B}(k, k-1) \mathbf{\Gamma}(k-1) \mathbf{B}^{\prime}(k, k-1)+\mathbf{Q}(k),
\end{aligned}
$$

where $\mathbf{A}$ and $\mathbf{B}$ are the Jacobian matrices of $\mathbf{f}(\cdot)$ with respect to $\overline{\mathbf{X}}$ and $\mathbf{U}, \mathbf{P}(k, k-1)$ is the covariance matrix of time update, and $\boldsymbol{\Gamma}$ and $\mathbf{Q}$ are the covariance matrices of the process noise and the input noise, respectively.

(2) Measurement update:

$$
\begin{aligned}
{[\mathbf{P}(k)]^{-1}=} & {[\mathbf{P}(k, k-1)]^{-1}+\mathbf{H}_{\mathrm{V}}^{\prime}(k)\left(\mathbf{R}_{\mathrm{V}}(k)\right)^{-1} \mathbf{H}_{\mathrm{V}}(k) } \\
& +s \cdot \mathbf{H}_{\mathrm{R}}^{\prime}(k)\left(\mathbf{R}_{\mathrm{R}}(k)\right)^{-1} \mathbf{H}_{\mathrm{R}}(k), \\
\mathbf{K}_{\mathrm{V}}(k)= & \mathbf{P}(k) \mathbf{H}_{\mathrm{V}}^{\prime}(k)\left(\mathbf{R}_{\mathrm{V}}(k)\right)^{-1}, \\
\mathbf{K}_{\mathrm{R}}(k)= & \mathbf{P}(k) \mathbf{H}_{\mathrm{R}}^{\prime}(k)\left(\mathbf{R}_{\mathrm{R}}(k)\right)^{-1}, \\
\widehat{\mathbf{X}}(k)= & \widehat{\mathbf{X}}(k, k-1)+\mathbf{K}_{\mathrm{V}}(k)\left[\mathbf{Z}_{\mathrm{V}}(k)-\mathbf{h}_{\mathrm{V}}(\widehat{\mathbf{X}}(k, k-1))\right] \\
& +\boldsymbol{s} \cdot \mathbf{K}_{\mathrm{R}}(k)\left[\mathbf{Z}_{\mathrm{R}}(k)-\mathbf{H}_{\mathrm{R}}(k)(\widehat{\mathbf{X}}(k, k-1))\right],
\end{aligned}
$$

where $\mathbf{P}(k)$ is the covariance matrix of measurement update. $\mathbf{K}_{\mathrm{V}}$ and $\mathbf{K}_{\mathrm{R}}$ represent the Kalman gains related with the measurement sources, i.e., DR sensors and preliminary positioning results, respectively. $\mathbf{R}_{\mathrm{V}}$ and $\mathbf{R}_{\mathrm{R}}$ are the corresponding covariance matrices of the measurement noises. $\mathbf{H}_{\mathrm{V}}$ is the Jacobian matrix of $\mathbf{h}_{\mathrm{V}}(\cdot)$. $s$ indicates the scale factor to achieve adaptability and the value of it is 0 or 1 .

From (21) and (24), it can be seen obviously that the measurement update stage can be executed by linear integration of individual measurement information. Due to such decentralized architecture, it is easy to exclude the preliminary positioning results when the information is failure or unavailable. The unavailability of RFID can be contributed such reasons:

(i) The detected tags are not enough, i.e., the number of tags is less than 4

(ii) The RFID observation time does not arrive (i.e., between consecutive transmit epochs)

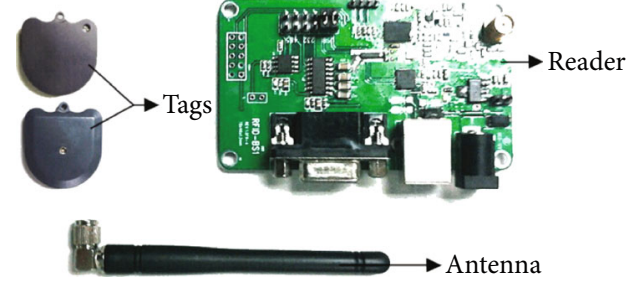

Figure 2: RFID hardware devices.

(iii) RFID may fail in adverse environments such as in a tunnel which may cause signal interruption or multipath interference

The RFID failure can be preliminary judged by the number of detected tags. If the number of detected tags drops below a certain threshold $N(=4)$, the RFID measurement information should be excluded from the measurement update stage. Moreover, to further guarantee the RFID failure can be detected reliably, we learn the fault diagnosis method of GPS and design a validation criterion [22] as follows.

$$
\begin{aligned}
\lambda= & {\left[\mathbf{Z}_{\mathrm{R}}(k)-\mathbf{H}_{\mathrm{R}}(k)(\mathbf{X} \wedge(k, k-1))\right]^{\prime} \cdot \mathbf{S}_{\mathrm{R}}^{-1}(k) } \\
& \cdot\left[\mathbf{Z}_{\mathrm{R}}(k)-\mathbf{H}_{\mathrm{R}}(k)(\widehat{\mathbf{X}}(k, k-1))\right] \leq \lambda_{\text {thres }},
\end{aligned}
$$

where $\mathbf{S}_{\mathrm{R}}(k)=\mathbf{H}_{\mathrm{R}}(k) \mathbf{P}(k, k-1) \mathbf{H}_{\mathrm{R}}^{\prime}(k)+\mathbf{R}_{\mathrm{R}}$. If $\lambda$ is less than or equal to $\lambda_{\text {thres}}$, it indicates that the preliminary results from RFID are considered to be effective and should be utilized, else if $\lambda$ is larger than $\lambda_{\text {thres}}$, it denotes that the preliminary positioning results are ineffective due to some reasons such as signal interruption or multipath interference, and the information should be excluded in the measurement update stage.

Considering such factors above, we could design the adaptive rules to judge the effectiveness of preliminary results from RFID and decide if the information is utilized in the measurement update stage, i.e., if (RFID observation time does not arrive) or $(N<4)$ or $\left(\lambda>\lambda_{\text {thres }}\right)$, $s=0$, else $s=1$.

\section{Experiments and Results}

To evaluate the proposed strategy, several field tests were conducted on the experimental vehicle. The sensor data were recorded via using a Buick Sail SRV vehicle which was equipped with RFID hardware devices sampled $1 \mathrm{~Hz}$ update rate, MEMSIC MEMS-based IMU VG440CA-200 inertial sensors sampled at $100 \mathrm{~Hz}, \mathrm{KVH} \mathrm{C} 100$ plus electronic compass with $1 \mathrm{~Hz}$ update rate, and a wheel speed sensor based on photoelectric encoder sampled at $1 \mathrm{~Hz}$.

The RFID devices, as shown in Figure 2, included one reader with an antenna and a number of active RFID tags. The RSS range of tag is normalized to $0-255$, and the maximum measured distance of the tag is $9 \mathrm{~m}$.

The MEMS-based IMU is shown in Figure 3. The specifications of the MEMS-based IMU can be found in Table 1. In this paper, only data from longitudinal and lateral accelerometers and yaw gyroscopes are used. 


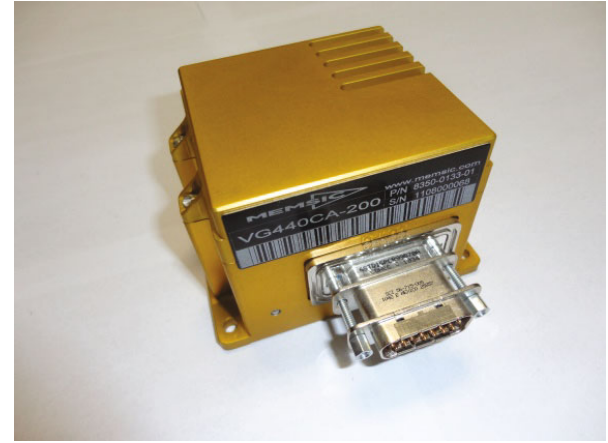

Figure 3: The MEMS-based IMU.

TABLE 1: MEMSIC IMU specifications (IMU VG440CA-200).

\begin{tabular}{lc}
\hline Gyroscope & \\
Range: roll, pitch, yaw & $\pm 10 \pi / 9 \mathrm{rad} / \mathrm{s}$ \\
Bias stability in-run & $<\pi \times 10^{-2} / 648 \mathrm{rad} / \mathrm{s}$ \\
Bias stability over temp & $<\pi \times 10^{-2} / 90 \mathrm{rad} / \mathrm{s}$ \\
Resolution & $<\pi \times 10^{-2} / 90 \mathrm{rad} / \mathrm{s}$ \\
Accelerometer & $\pm 4 * 10^{-3} \mathrm{~kg}$ \\
Range: $X / Y / Z$ & $<10^{-6} \mathrm{~kg}$ \\
Bias stability in-run & $<4 \times 10^{-6} \mathrm{~kg}$ \\
Bias stability over temp & $<5 \times 10^{-7} \mathrm{~kg}$ \\
Resolution &
\end{tabular}

The accuracies of other sensors $(1 \sigma)$ are $\pi / 360 \mathrm{rad}$ for the yaw angle of electronic compass and $0.05 \mathrm{~m} / \mathrm{s}$ for the longitudinal velocity of wheel speed sensor. Moreover, an accurate and reliable NovAtel SPAN-CPT system was used as a reference for quantitative comparison. The positioning accuracy of SPAN-CPT system was $0.01 \mathrm{~m}$ with GNSS observations and $0.02 \mathrm{~m}$ during $10 \mathrm{~s}$ outages.

During the field tests, all data from multiple sensors were gathered, and then, the strategy was verified utilizing the logged data.

5.1. Performance of Range Estimation. In the experiment, a RFID signal transmitting antenna was equipped onboard, and a number of active tags were placed on both sides of the road with known exact position.

Different scenes contain indoor, outdoor unobstructed environment, tunnel, and the simulated urban canyon environment, in which under the skyscrapers we model the correlation between RSS and range using ELM. For comparison, the propagation model-based algorithm utilizing Friis transmission equation is also investigated. Figure 4 gives the mean and standard deviation (STD) of estimation errors by the ELM and propagation model method, respectively. As an example, Figure 5 illustrates the measured normalized RSS for ranges between $0 \mathrm{~m}$ and $9 \mathrm{~m}$ in outdoor unobstructed environment. The range estimation results utilizing ELM and propagation model are also showed in Figure 5. The RSS has been measured in outdoor unobstructed conditions with range from $0.1 \mathrm{~m}$ to $2.0 \mathrm{~m}$ with an interval of $0.1 \mathrm{~m}$. For each point in Figure 5, 400 measurements of RSS from 4 different tags have been averaged.

It can be seen in Figures 4 and 5 that the ELM achieves better performance than the propagation model in different environments in terms of higher precision, higher generalization ability, and stronger adaptability, which can provide more accurate range information, i.e., about 50\% improvement, and then can enhance the performance of subsequent positioning algorithm.

5.2. Validation of Preliminary Positioning. To validate feasibility of the application of RFID for vehicle positioning, the preliminary positioning algorithm discussed above is evaluated through experiments firstly. Moreover, the multilateration method and standard EKF method are also investigated for comparison. The reference trajectories and the estimation results are shown in Figure 6, and the positioning errors are shown in Figure 7. Figure 8 gives the statistics of Euclidean distance errors (i.e., horizontal position errors) that contain the maximum value and the root mean square (RMS) value.

From Figure 8, we can find that the RMS error of the proposed AEKF algorithm is $5.12 \mathrm{~m}$, i.e., achieve $55 \%$ accuracy improvement compared to multilateration method and $28 \%$ improvement compared to standard EKF method. The max error of the proposed algorithm is obviously decreased to $8.13 \mathrm{~m}$ from the value $27.79 \mathrm{~m}$ of multilateration and $11.34 \mathrm{~m}$ of standard EKF method. Therefore, the accuracy of the proposed algorithm has remarkable improvement than that of the multilateration method and the standard EKF method. Compared with standard EKF, the proposed AEKF has better accuracy since it can adaptively adjust the measurement noise vector according to the changes of innovation vector. To further illustrate the reliability of the proposed $\mathrm{AEKF}$ in the scenario where certain tags are malfunctioning, some tags are assumed to fail to provide accuracy RSS information in the experiment, i.e., the noises of these tags are intentionally increased. The positioning results by using the standard EKF and the proposed AEKF are shown in Figure 9.

From Figure 9, we can find that the proposed AEKF can obtain better performance in the areas where some tags fail to provide reliable RSS information. The main reason is that if the noise from some tags is increased, the innovation may change adaptively according the changes, and then, the measurement noise of AEKF can be adaptively calculated to accommodate the changes in real time.

The proposed AEKF can achieve better preliminary positioning performance than the widely used multilateration method and the standard EKF method. However, there are also some deficiencies in the preliminary positioning utilizing AEKF, such as low output frequency and the accuracy is not high enough. In particular, from Figure 7, we can find that the errors are large when the vehicle is outside of the tag's coverage areas; it can be attributed that there are not enough tags detected by the reader in these areas, i.e., the number of tags is less than 4 . These problems can influence the 

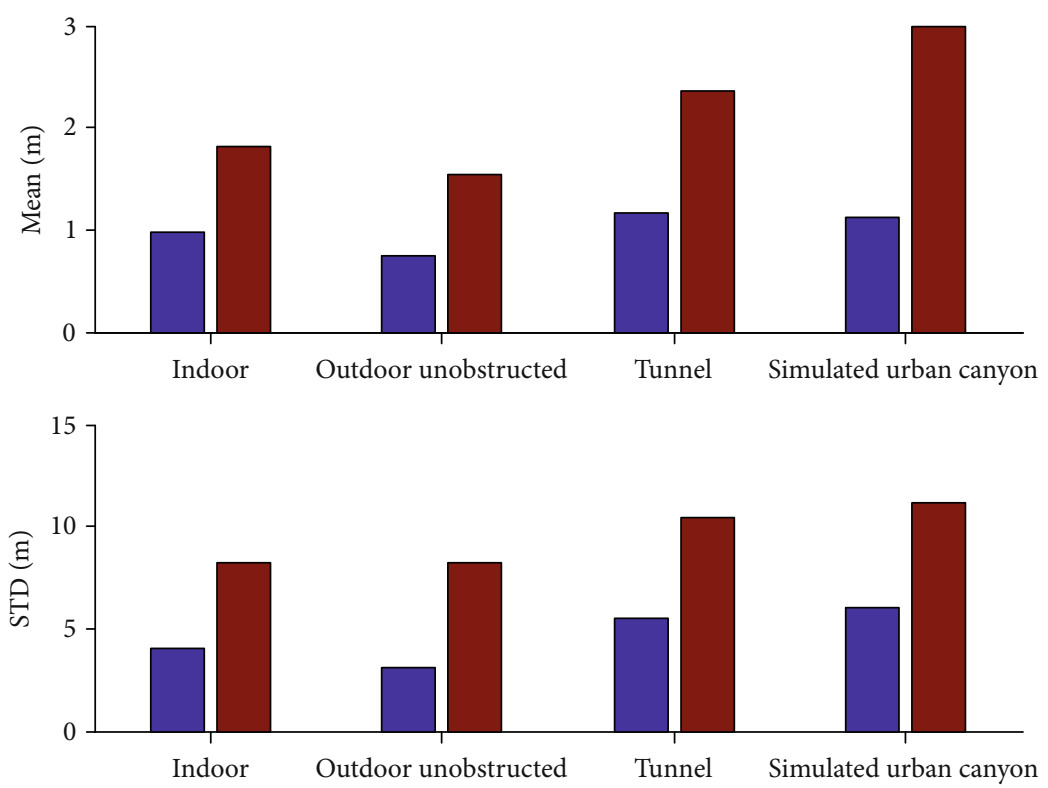

Different situations

ELM

Propagation model

Figure 4: The statistics of estimation errors.

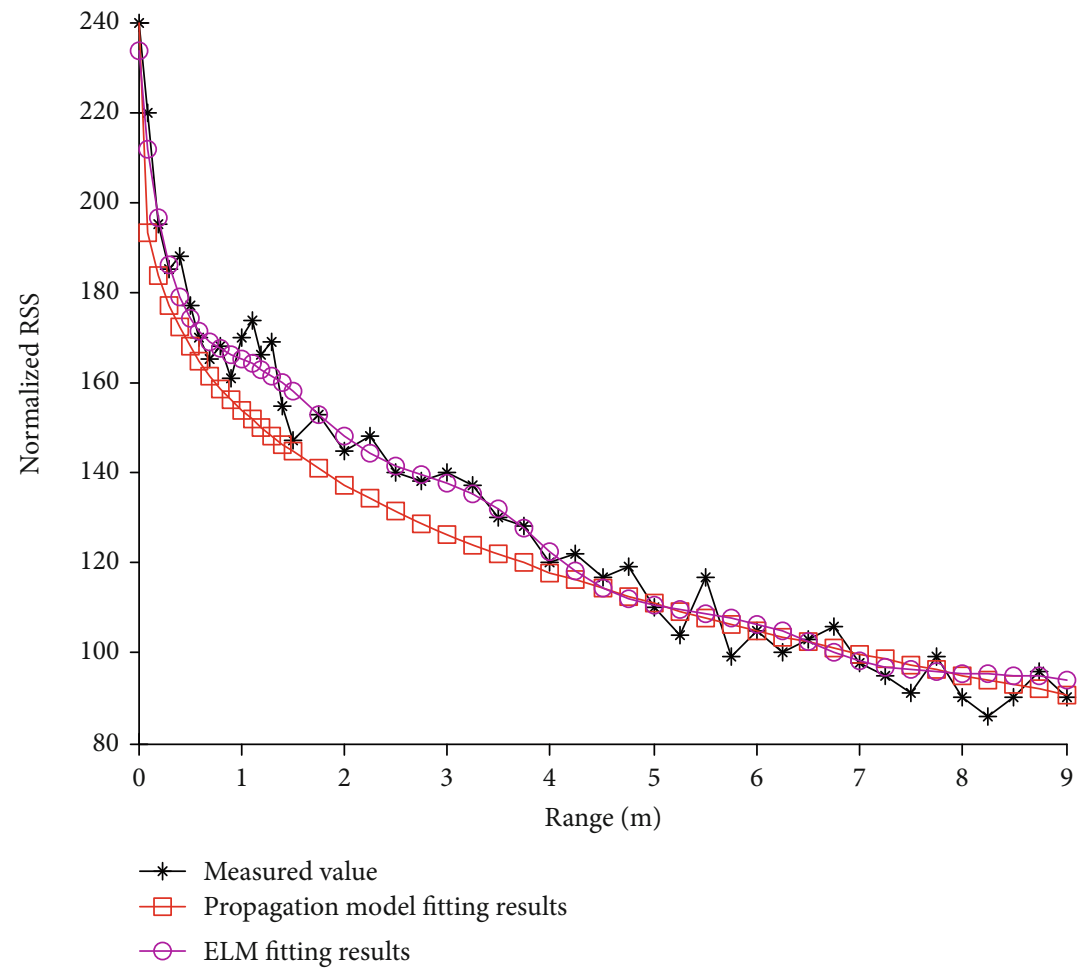

Figure 5: The fitting results of the relationship.

reliability of vehicle positioning and can be solved through the subsequent fusion positioning.

5.3. Performance of Fusion Positioning. To comprehensively illustrate the effective improvement of the proposed strategy, the scenario set of test contains the straight line situation and the curvilinear situation. For comparison, A DR-based algorithm which only uses the in-vehicle sensors to reckon the vehicle's position and the conventional EKF algorithm for fusion positioning are also investigated. 


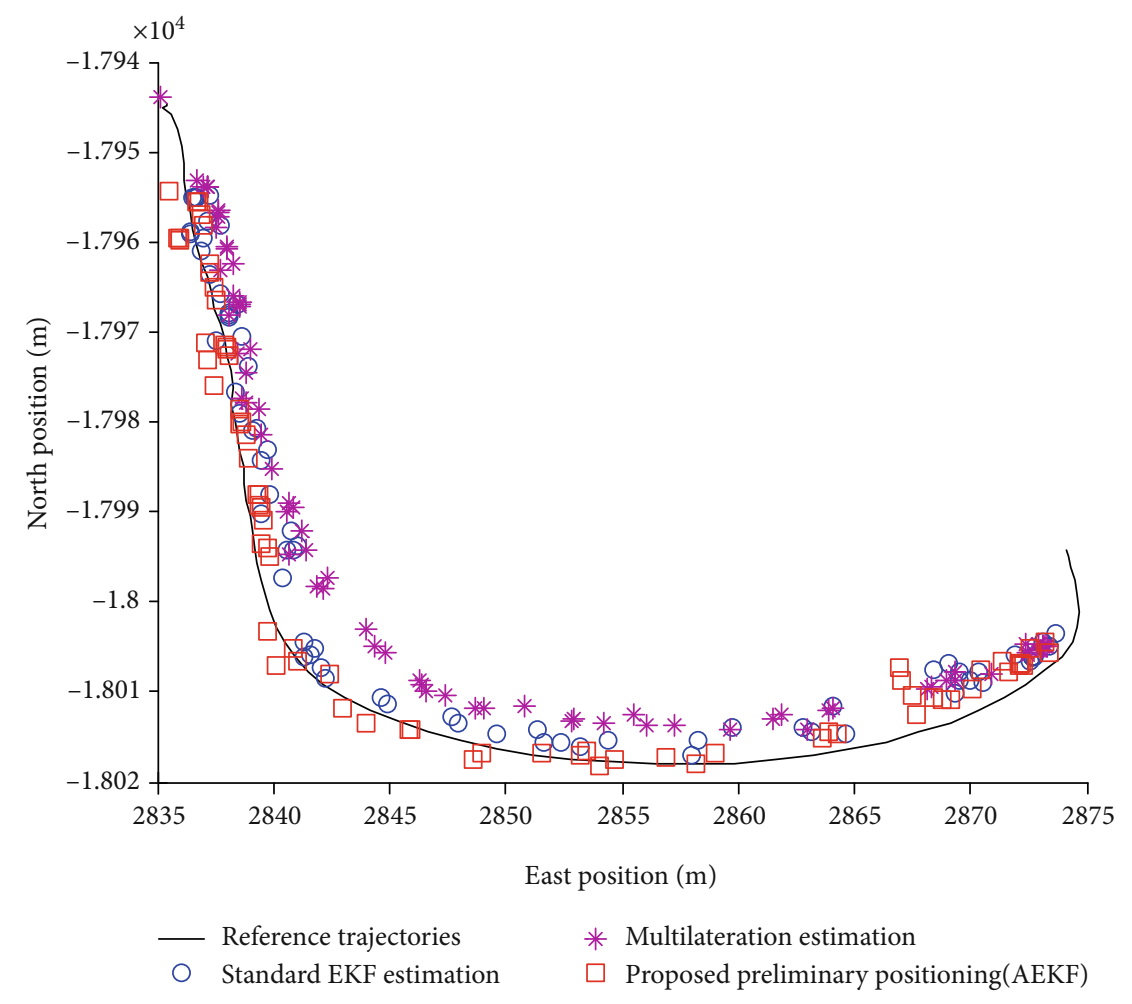

Figure 6: The vehicle trajectories.
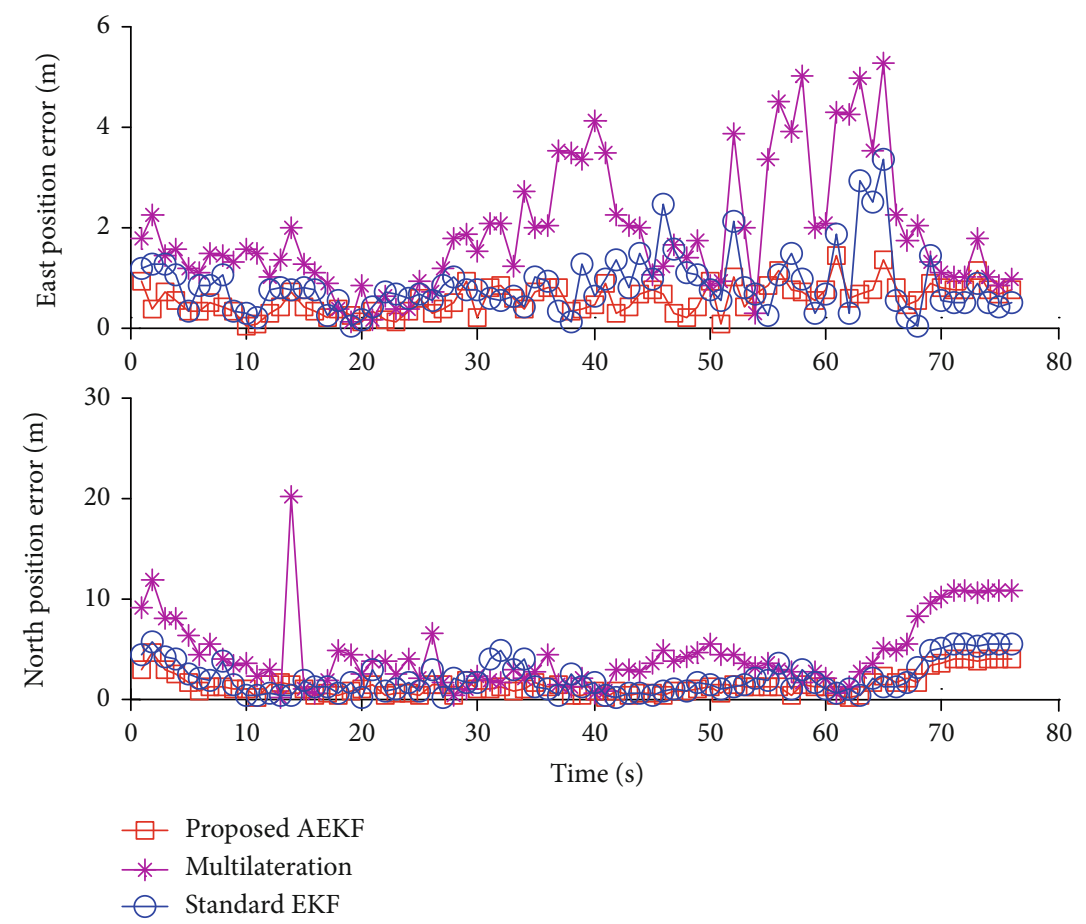

FIgURE 7: The east and north positioning errors.

In order to verify the adaptability of the algorithm in different typical motion patterns of the vehicle, a total of 21 tests in different driving modes, i.e., acceleration and deceleration motion in the straight line, curve motion with acceleration and deceleration, and lane change, have been carried out. The RMS of the Euclidean distance errors from 8 typical tests 


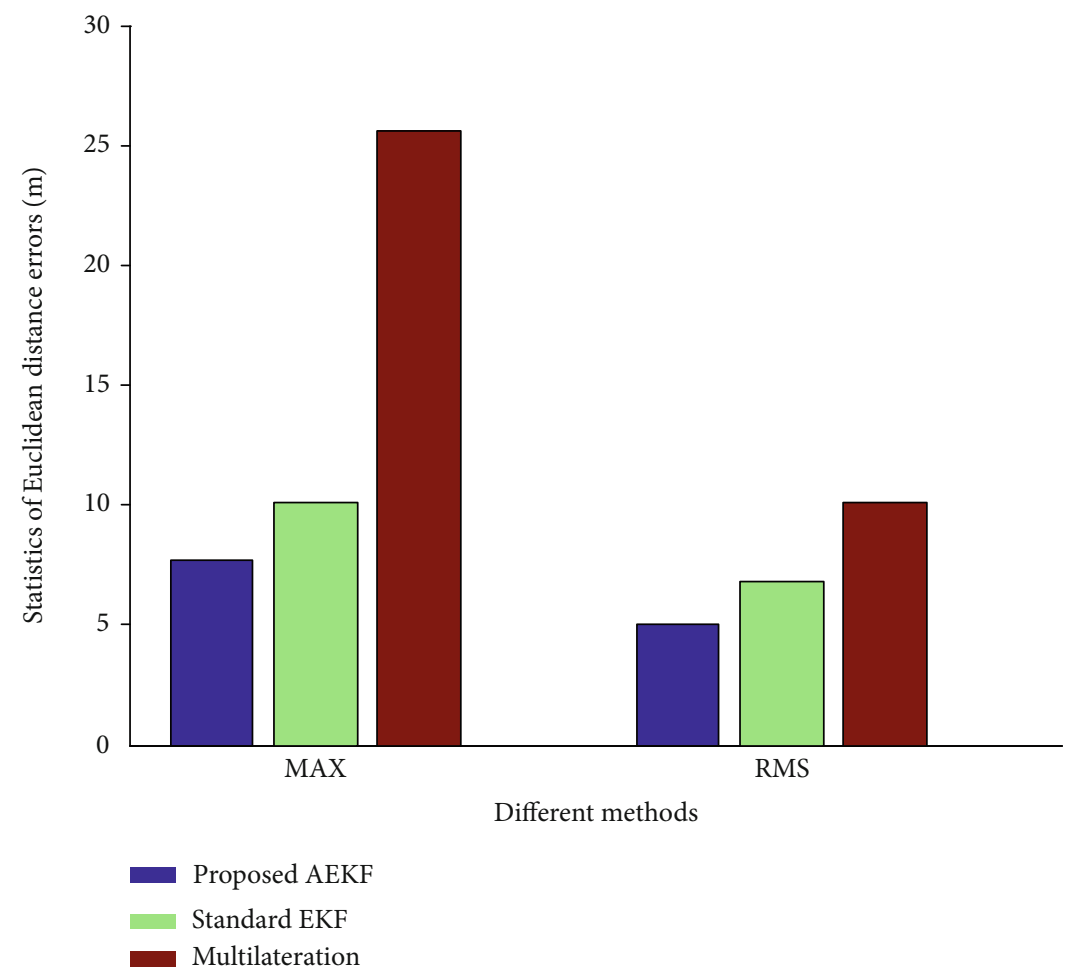

FIGURE 8: Statistics of Euclidean distance errors.

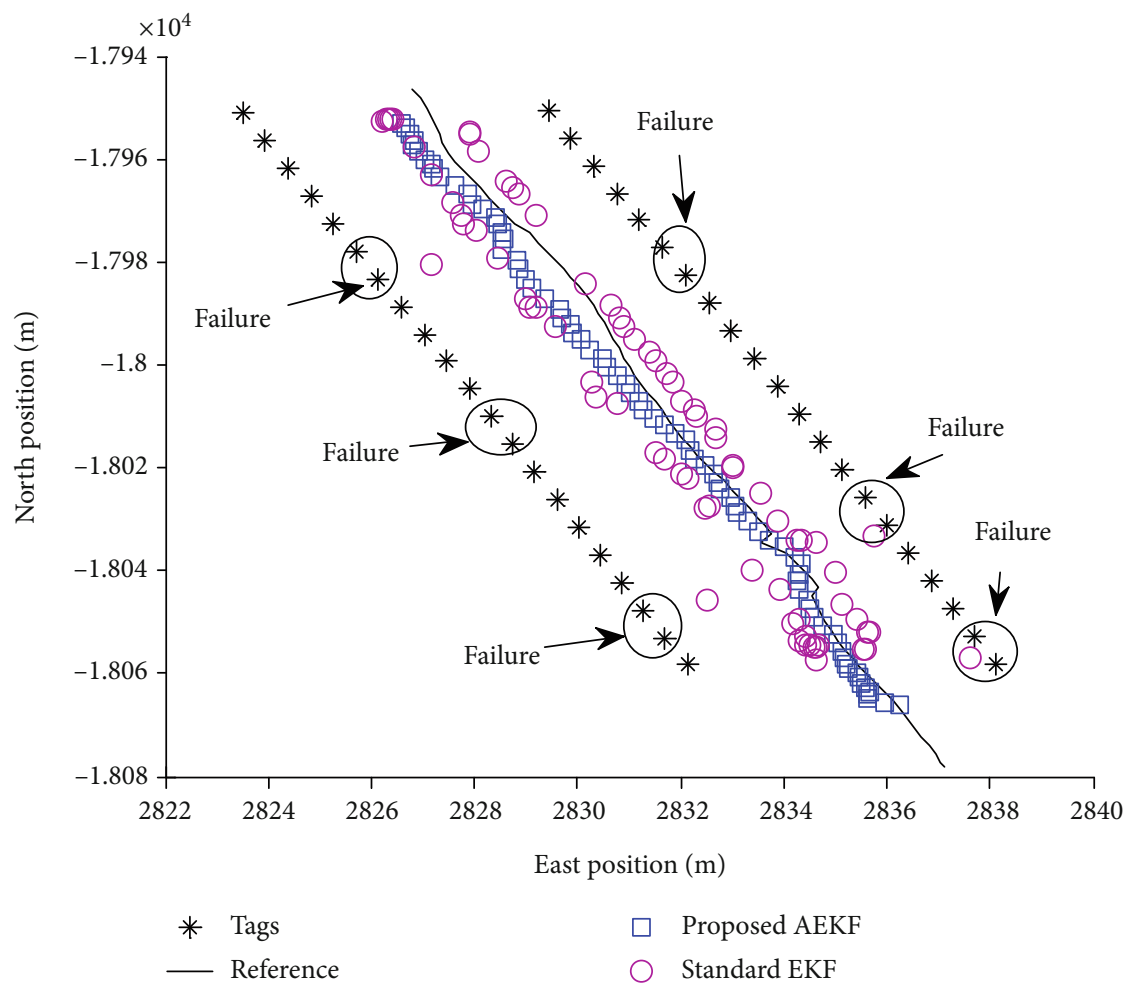

FIGURE 9: The estimation results in tag failure scenario.

which contain different motion patterns are summarized in Figure 10.

From Figure 10, we can find that the DR has the worst accuracy due to accumulative error and no correction infor- mation from position observation, and the proposed ADIFbased fusion algorithm achieves the optimal performance.

For brevity, we choose only one test as an instance since similar conclusions can be drawn from other 


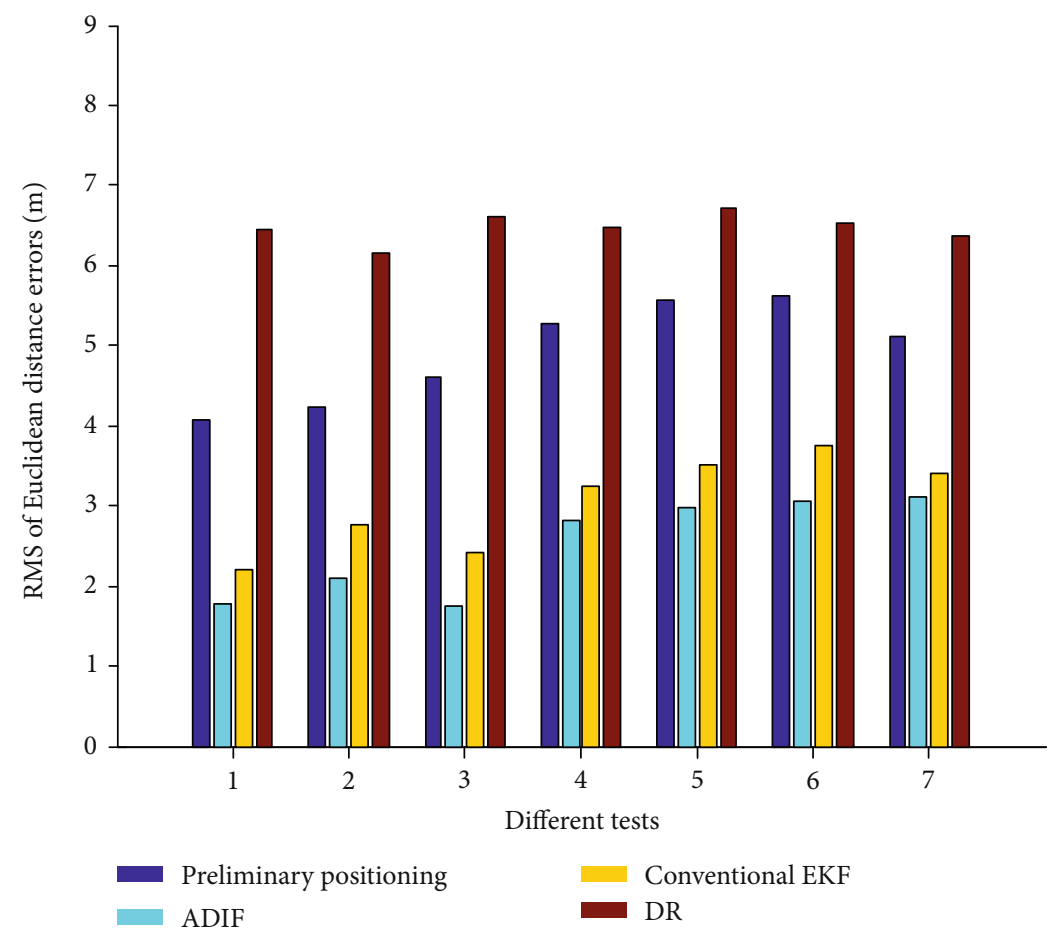

FIGURE 10: RMS of Euclidean distance errors in different tests.

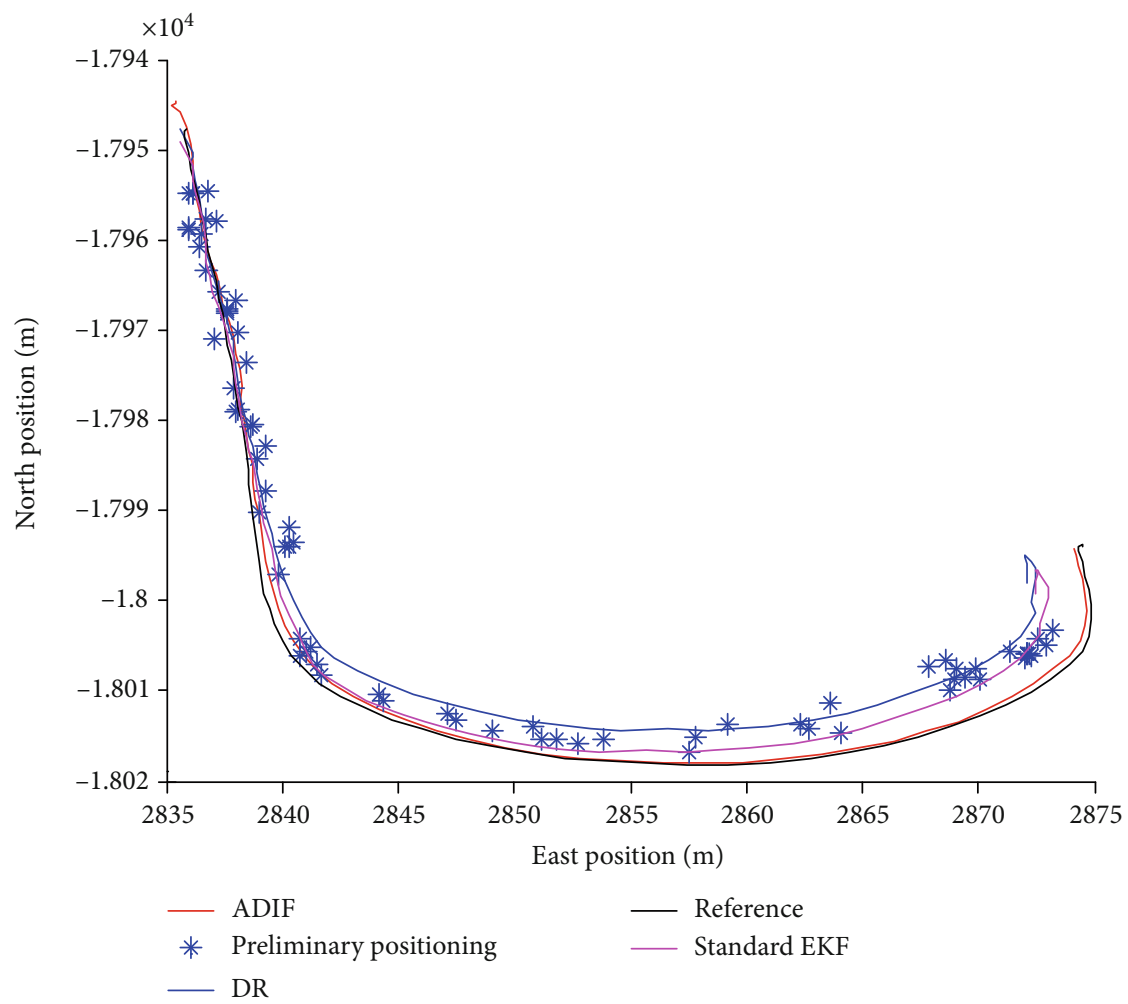

Figure 11: The vehicle trajectories.

experimental results. Figure 11 shows the trajectories of the selected test.

For four positioning algorithms, i.e., preliminary positioning, DR, ADIF-based fusion positioning, and standard EKF-based fusion positioning, Table 2 gives their performances. Figure 12 illustrates the east position errors of four algorithms as example.

From Table 2, it can be seen that the DR has the worst accuracy, i.e., both the RMS and the maximum values of its 
TABLE 2: The positioning performance of different methods.

\begin{tabular}{lcccc}
\hline \multirow{2}{*}{ Method } & \multicolumn{2}{c}{ Statistics of Euclidean distance errors (unit: m) } & \multicolumn{2}{c}{ Velocity output frequency (unit: Hz) } \\
& Max & RMS & No & 1 \\
Preliminary & 12.19 & 5.47 & Yes & 10 \\
DR & 11.14 & 7.21 & Yes & 10 \\
EKF & 7.71 & 4.17 & Yes & 10 \\
ADIF & 2.76 & 2.31 & & \\
\hline
\end{tabular}

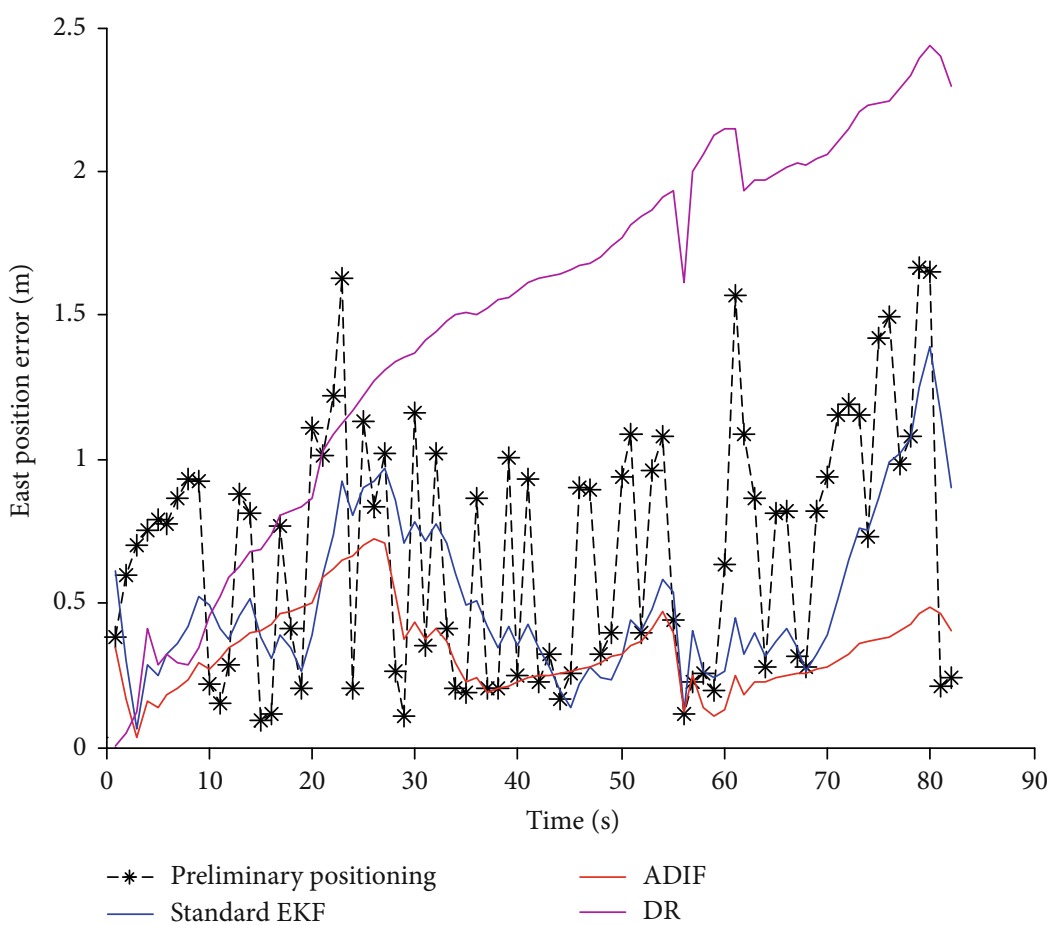

FIGURE 12: The east position errors of four algorithms.

Euclidean distance error are the largest. It can be attributed that the errors of DR algorithm will deteriorate over time. The preliminary positioning can achieve higher accuracy than DR. However, the max value of its Euclidean distance error is large. The reason is that the estimation results of the distances between tags and reader contain a lot of noise and sometimes even the tag failure.

From Table 2 and Figure 12, it is obvious that the positioning accuracy of ADIF-based fusion positioning and conventional EKF-based fusion positioning algorithms is improved obviously when compared with the preliminary positioning algorithm. For instance, the RMS value of ADIF error is decreased to $2.31 \mathrm{~m}$ from the value $5.47 \mathrm{~m}$ of preliminary positioning and the value $7.21 \mathrm{~m}$ of DR. It can be attributed that the more accurate and richer vehicle motion information is provided from the DR sensors, which can remarkably improve the positioning accuracy. Meanwhile, due to the improved vehicle motion model, ADIF-based and conventional EKF-based fusion positioning algorithms can adjust to the different vehicle operation status which contains straight motion and curvilinear motion.
Compared to EKF, the ADIF has better performance since there are inaccuracies of the noise description and unrealistic assumption of the model parameters in experiments. The max value of Euclidean distance error of ADIF is reduced from $7.71 \mathrm{~m}$ to $2.76 \mathrm{~m}$, i.e., about $65 \%$ accuracy improvement over conventional EKF. The main reason is that the decentralized architecture and the adaptive rules can remarkably enhance the performance, especially under the circumstance when the information from preliminary positioning is failure or unavailable. For intuitive illustration of the advantages of ADIF, the max values of Euclidean distance errors by utilizing standard EKF and ADIF are shown in Figure 13. For comparison, the max values of Euclidean distance errors of preliminary positioning are also given.

From Figure 13, we can find that the max values of preliminary positioning errors are very large at the start and end time of experiment. In these periods, the vehicle is outside or just enters the tag's coverage areas, and the number of detected tags is small, i.e., less than 4 . The results of standard EKF can get some improvements; however, the correction effect is poor and the problem still exists. It can be 


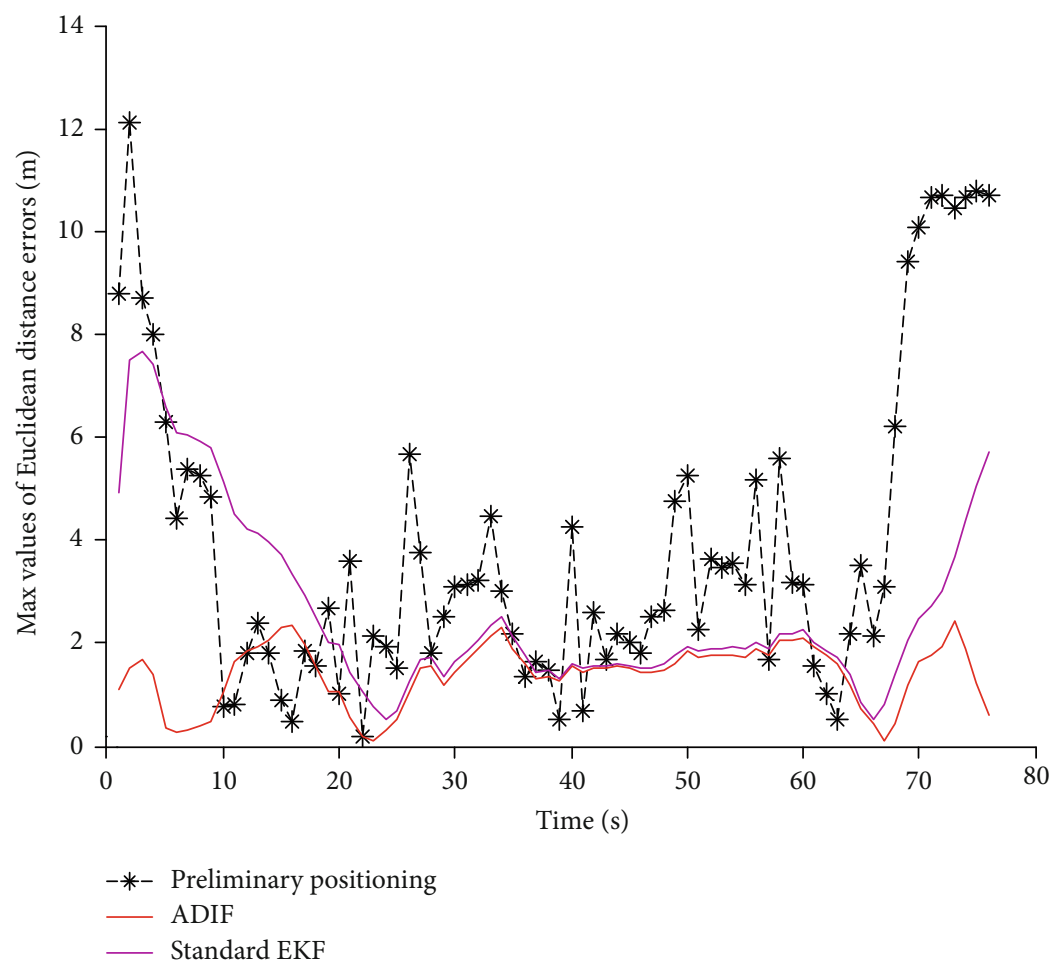

FIGURE 13: The max values of Euclidean distance errors.

obviously seen in Figure 13; the ADIF algorithm can solve this problem, i.e., the $\max$ values of positioning errors are small at any time. It can be attributed that if the number of detected tags is less than 4, the preliminary positioning results are shielded according to the adaptive rules, and the decentralized filtering algorithm can guarantee that the final fusion positioning results come from DR without pollution of false preliminary positioning results.

Therefore, the proposed ADIF-based fusion positioning algorithm achieves the best accuracy and reliability among the other four methods. Compared with the preliminary positioning algorithm in which only RFID is used, the ADIF-based fusion positioning algorithm provides obviously performance improvement, e.g., over 58\%. Its positioning frequency rose to $10 \mathrm{~Hz}$ from $1 \mathrm{~Hz}$ of preliminary positioning, and the velocity information can be obtained.

Compared with low-cost GPS, which is the most popularly used technology for vehicle positioning, the proposed strategy has the approximation accuracy with higher frequency. Therefore, when GPS is completely unavailable, the strategy can satisfy the demands for the vehicle's locatebased applications.

\section{Conclusions}

To achieve accurate and reliable positioning in completely GPS-denied environments, a fusion strategy for vehicle positioning is proposed. This strategy employs two-step approaches, namely, RFID is employed to locate the vehicle, replacing the GPS, to achieve preliminary positioning, and then, in-vehicle DR sensors are introduced to integrate with RFID to achieve fusion positioning.
In the strategy, a two-level filtering method is proposed to achieve preliminary and fusion positioning. First, the RSS of RFID is employed as an indicator to locate the vehicle preliminarily, and the ELM algorithm is developed to obtain the range between RFID tag and reader. Then, the firstlevel AEKF with better ability to accommodate the uncertainties in the observation noise description is designed to achieve preliminary positioning. Further, the second-level ADIF algorithm is developed to realize the fusion positioning. Rather than conventional EKF, the ADIF with decentralized architecture is developed; it could isolate the RFID failure under the proposed adaptive rules. Experiments were performed to verify the effectiveness of both preliminary and fusion positioning algorithms comprehensively. The experimental results show that there are more obvious advantages of the proposed strategy than other methods when GPS is unavailable.

\section{Data Availability}

The experimental data used to support the findings of this study are available from the corresponding author upon request.

\section{Conflicts of Interest}

The authors declare no conflict of interest.

\section{Acknowledgments}

This research was funded by the National Natural Science Foundation of China, grant number 61801227; the Qinglan 
Project of Jiangsu Province of China, grant number QLGC-2020; the Natural Science Foundation of the Jiangsu Higher Education Institutions of China, grant numbers 18KJB413007, 18KJB510022 and 19KJD420002; the Jiangsu Province Industry-University-Research Cooperation Project, grant number BY2019078; the key program of Nanjing Xiaozhuang University, grant number 2017NXY12; and the Open Project of Key Laboratory of Road Traffic Safety Ministry of Public Security, grant number 2019ZDSYSKFKT06.

\section{References}

[1] H. H. Liu and C. Liu, "Implementation of Wi-Fi signal sampling on an android smartphone for indoor positioning systems," Sensors, vol. 18, no. 1, p. 3, 2018.

[2] Y. Dobrev and P. Gulden, "An Indoor Positioning System Based on Wireless Range and Angle Measurements Assisted by Multi-Modal Sensor Fusion for Service Robot Applications," IEEE Access, vol. 6, pp. 69036-69052, 2018.

[3] J. Talvitie and M. Renfors, "Distance-Based Interpolation and Extrapolation Methods for RSS-Based Localization With Indoor Wireless Signals," IEEE transactions on vehicular technology, vol. 64, no. 4, pp. 1340-1353, 2015.

[4] I. Sharp and K. Yu, "Enhanced least-squares positioning algorithm for indoor positioning," IEEE Transactions on Mobile Computing, vol. 12, no. 8, pp. 1640-1650, 2013.

[5] J. Zhou and J. Shi, "RFID localization algorithms and applications-a review," Journal of intelligent manufacturing, vol. 20, no. 6, pp. 695-707, 2009.

[6] X. Song and X. Li, "A fusion strategy for reliable vehicle positioning utilizing RFID and in-vehicle sensors," Information Fusion, vol. 31, pp. 76-86, 2016.

[7] C. Shen and Y. Zhang, "Dual-optimization for a MEMSINS/GPS system during GPS outages based on the cubature Kalman filter and neural networks," Mechanical Systems and Signal Processing, vol. 133, p. 10622, 2019.

[8] X. Li and W. Chen, "Multi-sensor fusion methodology for enhanced land vehicle positioning," Information Fusion, vol. 46, pp. 51-62, 2018.

[9] C. Shen and Y. Zhang, "Seamless GPS/inertial navigation system based on self-learning square-root cubature Kalman filter," IEEE Transactions on Industrial Electronics, 2020.

[10] L. Byung-Hyun and S. Jong-Hwa, "GPS/DR error estimation for autonomous vehicle localization," Sensors, vol. 15, no. 8, pp. 20779-20798, 2015.

[11] T. N. Van and T. C. Duc, "Application of street tracking algorithm in an INS/GPS integrated navigation system," IETE Journal of Research, vol. 61, no. 3, pp. 1-9, 2015.

[12] K. Jo and K. Chu, "Interacting multiple model filter-based sensor fusion of GPS with in-vehicle sensors for real-time vehicle positioning," IEEE Transactions on Intelligent Transportation Systems, vol. 13, no. 1, pp. 329-343, 2012.

[13] S. Nisar and I. Ullah, "Non-linear filtering techniques for high precision GPS applications," in International Conference on Communication, pp. 276-280, Islamabad, Pakistan, 2017.

[14] H. Ahmed, I. Ullah, U. Khan et al., "Adaptive filtering on GPSaided MEMS-IMU for optimal estimation of ground vehicle trajectory," Sensors, vol. 19, no. 24, p. 5357, 2019.

[15] D. Bhatt and P. Aggarwal, "A novel hybrid fusion algorithm to bridge the period of GPS outages using low-cost INS," Expert Systems with Applications, vol. 41, no. 5, pp. 2166-2173, 2014.
[16] X. Li and Q. Xu, “A reliable fusion positioning strategy for land vehicles in GPS-denied environments based on low-cost sensors," IEEE Transactions on Industrial Electronics, vol. 64, no. 4, pp. 3205-3215, 2017.

[17] N. Davari and A. Gholami, "An direct Kalman algorithm to navigation Performance," IEEE Sensors Journal, vol. 17, no. 4, pp. 1061-1068, 2017.

[18] M. Rafie and T. Niknam, "Probabilistic forecasting of hourly electricity price by generalization of ELM for usage in improved wavelet neural network," IEEE Transactions Industrial Electronics, vol. 13, no. 1, pp. 71-79, 2017.

[19] F. Lazzari, A. Buffi, P. Nepa, and S. Lazzari, "Numerical Investigation of an UWB Localization Technique for Unmanned Aerial Vehicles in Outdoor Scenarios," IEEE Sensors Journal, vol. 17, no. 9, pp. 2896-2903, 2017.

[20] F. Ghaleb and A. Zainal, "Improved vehicle positioning algorithm using enhanced innovation-based adaptive Kalman filter," Pervasive and Mobile Computing, vol. 40, pp. 139-155, 2017.

[21] K. Tashfeen and A. Mohamed, "An enhanced error model for EKF-based tightly-coupled integration of GPS and land vehicle's motion sensors," Sensors, vol. 15, no. 9, pp. 2426924296, 2015.

[22] J. Y. Lee, H. K. Lee, and H. S. Kim, "Detection of multiple faults in single-frequency differential GPS measurements," IET Radar, Sonar \& Navigation, vol. 6, no. 8, pp. 697-707, 2012. 